\title{
Quantum control of isomerization by robust navigation in the energy spectrum
}

\author{
G. E. Murgida, ${ }^{1, a)}$ F. J. Arranz, ${ }^{2, b)}$ and F. Borondo ${ }^{3,4, c)}$ \\ ${ }^{1}$ Centro Atómico Constituyentes, GIyA, CNEA, San Martín, and Consejo Nacional de Investigaciones \\ Científicas y Técnicas, C1033AAJ Buenos Aires, Argentina \\ ${ }^{2}$ Grupo de Sistemas Complejos, Universidad Politécnica de Madrid, 28040 Madrid, Spain \\ ${ }^{3}$ Departamento de Química, Universidad Autónoma de Madrid, Cantoblanco, 28049 Madrid, Spain \\ ${ }^{4}$ Instituto de Ciencias Matemáticas (ICMAT), Cantoblanco, 28049 Madrid, Spain
}

(Received 17 August 2015; accepted 13 November 2015; published online 3 December 2015)

\begin{abstract}
In this paper, we present a detailed study on the application of the quantum control technique of navigation in the energy spectrum to chemical isomerization processes, namely, $\mathrm{CN}-\mathrm{Li} \leftrightarrows$ $\mathrm{Li}-\mathrm{CN}$. This technique is based on the controlled time variation of a Hamiltonian parameter, an external uniform electric field in our case. The main result of our work establishes that the navigation involved in the method is robust, in the sense that quite sizable deviations from a pre-established control parameter time profile can be introduced and still get good final results. This is specially relevant thinking of a experimental implementation of the method. (C) 2015 AIP Publishing LLC. [http://dx.doi.org/10.1063/1.4936424]
\end{abstract}

\section{INTRODUCTION}

New technologies in femtosecond laser pulses have made possible an increasing number of successful strategies to manipulate and control atoms, nuclei, electrons, molecules and other quantum systems. This includes closed loop methods characterized by periodic feedback measures to correct the control parameters. ${ }^{1-5}$ Decoherence is usually a limitation for quantum control, ${ }^{6}$ but recent technological advances have increased tremendously the coherence time up to several seconds for electron-spin ${ }^{7}$ or minutes in the case of some nuclear-spin. ${ }^{8}$ Moreover, there are also decoherent quantum control strategies, where coherence is destroyed in order to improve the controllability of the system, ${ }^{9-11}$ this allows to achieve states that cannot be accessed by unitary evolution. Measurement, manipulation, and computation of quantum information can be counted among some of the most important goals of quantum control. At present, the more promising candidates for a future quantum computer technology ${ }^{12}$ are electrodynamics cavities (QED), ion and neutral atom traps, quantum dots, optical systems, superconductor interference devices (SQUID), and nuclear magnetic resonance (NMR) setups. Among the experimental results obtained in recent years, coherent control of spin state of individual electrons ${ }^{13-15}$ and nuclei, ${ }^{16-18}$ generation of entangled states between electrons or photons, ${ }^{19}$ strong coupling and entanglement of quantum dots with photons, ${ }^{20-24}$ creation of artificial molecules using quantum dots, ${ }^{25,26}$ and universal control of charge qubits in quantum dots ${ }^{27}$ deserve special mention.

In the last two decades, several successful experimental techniques to manipulate molecules, such as infrared (IR)

\footnotetext{
a)Electronic mail: murgida@tandar.cnea.gov.ar

b)Electronic mail: fj.arranz@upm.es

c)Electronic mail: f.borondo@uam.es
}

resonant radiation, ${ }^{28-32}$ stimulated Raman adiabatic passage (STIRAP), ${ }^{33}$ and counter-diabatic fields, ${ }^{34}$ have allowed to populate specific vibrational states, to break or excite selected bonds, to promote isomerization, and to control the quantum dynamics of molecules. ${ }^{35-37}$ Isomerization and dissociation experiments using ultrafast IR pulses and feedback-based strategies were successfully applied to different molecules such as, for example, acetylene ${ }^{38}$ and $\mathrm{HCo}(\mathrm{CO})_{4}{ }^{39}$ While these isomerization experiments, and others recently proposed, ${ }^{34,40}$ employ pulses of tens or hundreds of cycles, other works propose to control nuclear dynamic and isomerization using only one very strong tailored pulse in the femtoseconds scale. ${ }^{41-43}$ In a recent theoretical work, Pellouchoud and $\mathrm{Reed}^{41}$ propose isomerizing a $\mathrm{Li}-\mathrm{CN}$ molecule using a single-cycle pulse of an electric field, whose shape design is based on the classical dynamics of the nuclei under a density-functional-theory (DFT)-computed field-dependent potential surface. This last method requires an accurate shaped very strong pulse with maximum values of the order of $1 \mathrm{~V} / \AA$. Nevertheless, recent improvements on sources of strong terahertz $(\mathrm{THz})$ pulses, allowing peaks of $0.2-0.4 \mathrm{~V} / \AA^{4},{ }^{43-45}$ suggest the plausibility of this strategy in a near future.

Recently, we proposed a strategy to isomerize $\mathrm{Li}-\mathrm{CN}$ using a tailored electric field pulse, ${ }^{46,47}$ where the quantum dynamics for the nuclei is considered. This method is based on the knowledge of the vibrational energy spectrum as a function of the electric field (adiabatic curves), and then, a time dependent electric field profile is designed in order to navigate the level curves using an optimized sequence of diabatic and adiabatic transitions leading to the desired final state. We showed that using this control method, a high probability of reaching the final state $(\sim 99 \%)$ can be achieved, and moreover, this requires electric field intensities weaker than those in the above mentioned ultrafast method (less 
than $0.13 \mathrm{~V} / \AA$, i.e., $3 \cdot 10^{-3}$ a.u.). However, the high accuracy needed to shape the optimized pulse can, in principle, represent a strong obstacle for its experimental implementation and, in addition, small variations in the shape or timing of the electric pulse can decrease significantly the efficiency of the method. In order to make possible a realistic implementation of the method for the $\mathrm{CN}-\mathrm{Li} \rightarrow \mathrm{Li}-\mathrm{CN}$ isomerization, we show here that a robust path in the spectrum can be found that, when used in the navigation, leads to a much simpler electric field time profile, actually consisting of only two linear pieces. Although this simplified shape of the electric pulse is not optimal, and then the final transition probability is reduced, it still has a high efficiency of $97 \%$. Moreover, we show that this control strategy is robust, in the sense that sizable variations in the slopes of the electric field profile do not significantly reduce the final transition probability.

The organization of the paper is as follows. In Sec. II, we introduce the LiCN molecular system, the model employed, and the numerical methods used in our calculation, which include a more accurate effective potential energy than that employed in our previous paper. ${ }^{47}$ Section III focuses on the control strategy. There we describe the path and the electric field time profiles derived from our calculations. The numerical results and the robustness of the control path are also analyzed here. Finally, our concluding remarks are summarized in Sec. IV. In Appendix A, we check the validity of the rotationless motion assumed in our model, and in Appendix B, we check the robustness of our results against the experimental inaccuracies in the alignment angle.

\section{SYSTEM DESCRIPTION AND CALCULATIONS}

\section{A. Isomerizing system Hamiltonian}

The $\mathrm{Li}-\mathrm{CN}$ molecule is a triatomic floppy molecule, ${ }^{48,49}$ within a class which includes, for example, $\mathrm{H}-\mathrm{CN},{ }^{50}$ $\mathrm{Na}-\mathrm{CN},{ }^{48} \mathrm{~K}-\mathrm{CN},{ }^{48,51} \mathrm{H}_{3} \mathrm{C}-\mathrm{CN},{ }^{52} \mathrm{H}-\mathrm{CP},{ }^{53}$ and the $\mathrm{H}-\mathrm{O}_{2}$ radical,${ }^{54}$ or the van der Waals complexes,${ }^{55}$ such as He-FH, $\mathrm{He}-\mathrm{ClH}, \mathrm{He}-\mathrm{BrH}$, and $\mathrm{He}-\mathrm{CNH}$.

In the case of $\mathrm{LiCN}$, the floppy motion corresponds to the $\mathrm{Li}-\mathrm{C}-\mathrm{N}$ bending, which is highly coupled to the $\mathrm{Li}-\mathrm{CN}$ stretching, but highly uncoupled (adiabatically) from the high frequency mode $\mathrm{C}-\mathrm{N}$. This makes possible to accurately decompose the complete vibrational dynamics in a $2+1$ model, ignoring the latter degree of freedom. ${ }^{56}$ The corresponding rotationless vibrational Hamiltonian function $H_{\mathrm{v}}$ is given, in Jacobi coordinates, by ${ }^{57}$

$$
H_{\mathrm{v}}=\frac{P_{R}^{2}}{2 \mu_{1}}+\left(\frac{1}{\mu_{1} R^{2}}+\frac{1}{\mu_{2} r_{\mathrm{eq}}^{2}}\right) \frac{P_{\theta}^{2}}{2}+V(R, \theta),
$$

where $r_{\text {eq }}$ is the fixed $\mathrm{C}-\mathrm{N}$ equilibrium distance, $R$ is the length between the $\mathrm{C}-\mathrm{N}$ center of mass and the $\mathrm{Li}$ atom $\left(\begin{array}{l}\mathrm{C} \\ \mathrm{N}\end{array} \rightarrow \mathrm{Li}\right)$, and $\theta$ is the angle formed by these two directions, with $\theta=0$ corresponding to the linear configuration $\mathrm{Li}-\mathrm{CN}$, and $\theta=\pi$ rad to $\mathrm{CN}-\mathrm{Li}$. The associate conjugate momenta are $P_{R}$ and $P_{\theta}$, respectively, with reduced masses: $\mu_{1}$ $=m_{\mathrm{Li}}\left(m_{\mathrm{C}}+m_{\mathrm{N}}\right) /\left(m_{\mathrm{Li}}+m_{\mathrm{C}}+m_{\mathrm{N}}\right)$ and $\mu_{2}=m_{\mathrm{C}} m_{\mathrm{N}} /\left(m_{\mathrm{C}}+m_{\mathrm{N}}\right)$, $m_{X}$ being the involved atomic masses. Function $V(R, \theta)$ is the potential energy for the interaction between the $\mathrm{Li}$ atom and the $\mathrm{CN}$ dimer. Notice that Hamiltonian (1) models the isomerization reaction $\mathrm{CN}-\mathrm{Li} \leftrightarrows \mathrm{Li}-\mathrm{CN} .{ }^{58}$

The potential energy surface $V(R, \theta)$ is taken as the $a b$ initio quantum calculations of Essers et al. fitted to an expansion in Legendre polynomials, ${ }^{49}$ and it is represented in Fig. 1(a) in the form of a contour plot. As can be seen, this function has two minima: A relative one at $\theta=0$ and another, which is absolute, at $\theta=\pi \mathrm{rad}$. Both correspond to linear isomers, which are separated by a saddle point barrier, and connected by the minimum energy path plotted in thick blue line in the figure.

\section{B. Control Hamiltonian}

The LiCN molecule is polar, thus presenting a permanent dipole moment. Actually, when all possible configurations of the system $\mathrm{CN}-\mathrm{Li} / \mathrm{Li}-\mathrm{CN}$ are considered, this dipole moment is represented by a vector field $\mathbf{p}(R, \theta)$. Accordingly, one can interact with the system, e.g., in order to control the isomerization, simply by placing it in an external fixed electric field $\mathcal{E}$. In this case, an additional term in the Hamiltonian appears

$$
H_{\mathrm{c}}=H_{\mathrm{v}}-\mathbf{p} \cdot \mathcal{E} .
$$

One can imagine now using molecular rotation control ${ }^{59}$ and molecular alignment techniques ${ }^{60-62}$ to align polar molecules, and then orientate our isomerizing system in such a way that the electric field $\mathcal{E}$ is parallel to the $\mathrm{C} \leftarrow \mathrm{N}$ bond. Under these conditions, the external field intensity $\mathcal{E}$ can be used as the control parameter for the reaction. In our calculations, it is assumed that this alignment is perfect, something which is obviously not true in actual experimental situations.
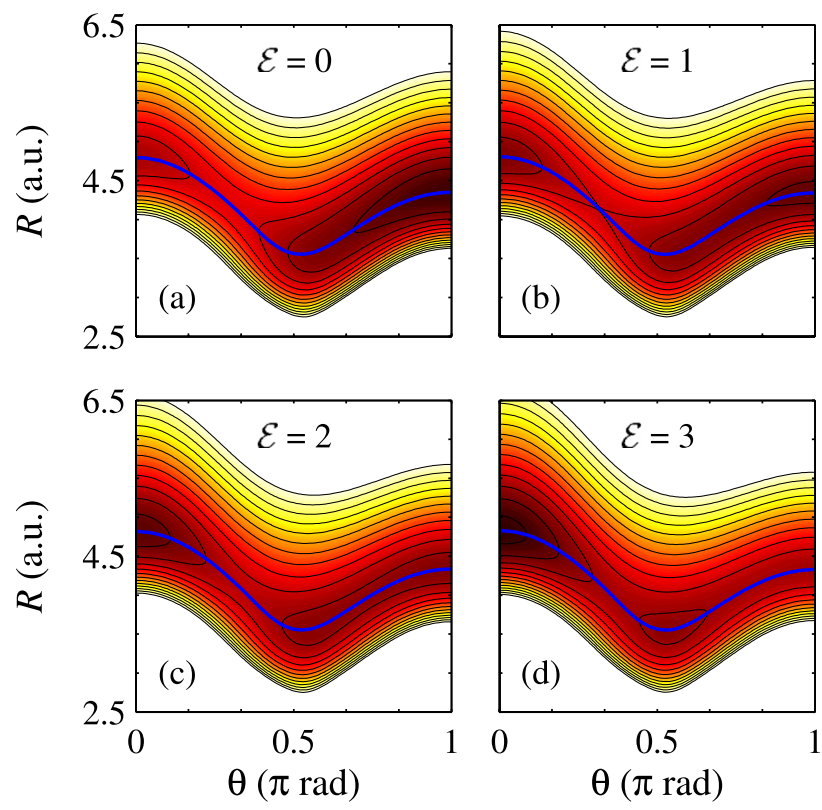

FIG. 1. Effective potential energy function $V_{\text {eff }}(R, \theta)$, as defined in Eq. (3) for the $\mathrm{CN}-\mathrm{Li} / \mathrm{Li}-\mathrm{CN}$ isomerizing system subjected to an electric field parallel to the $\mathrm{C} \leftarrow \mathrm{N}$ bond. The magnitude of the field intensity $\mathcal{E}\left(\times 10^{-3}\right.$ a.u. $)$ is indicated in each panel. Contours are plotted every $1000 \mathrm{~cm}^{-1}$. The minimum energy path connecting the two isomer wells has also been included in thick blue line. 
The effect on our study of considering the experimental inaccuracies will be discussed in Appendix B, at the end of the paper.

Furthermore, the extra term in Eq. (2) can be added to the potential energy function to define a new effective potential as

$$
V_{\mathrm{eff}}(R, \theta)=V(R, \theta)-\mathbf{p} \cdot \boldsymbol{\varepsilon} .
$$

In our case, we take the dipole moment vector function $\mathbf{p}(R, \theta)$ as the ab initio quantum calculations of Brocks et al. ${ }^{63}$ fitted by us to an expansion in Legendre polynomials. The corresponding effective potential for three different values of the external electric field intensity is presented in Figs. 1(b)-1(d). As can be seen, as $\mathcal{E}$ increases, the most stable well at $\theta=\pi$ rad becomes a saddle, unstable with regard to the isomerization, while at the same time, the well at $\theta=0$ progressively increases in stability. This result can be rationalized taking into account the sign in the interaction in Eq. (3) and the relative geometry between field and $\mathrm{CN}$ fragment which we have assumed. Notice also the emergence of a new well at $\theta \approx \pi / 2 \mathrm{rad}$, most prominent at $\mathcal{E}=3 \cdot 10^{-3}$ a.u., which corresponds to a triangular configuration. As we will describe in more detail later, this new well is very relevant in the isomerization control process: Actually, if we start at $\mathcal{E}(t=0)$ from an initial probability density localized in the well at $\theta=\pi \mathrm{rad}$ $(\mathrm{CN}-\mathrm{Li}$ isomer), it can be "shepherded" by means of a suitable time evolution of the increasing electric field $\mathcal{E}(t)$ to the well at $\theta=0$, corresponding to the $\mathrm{Li}-\mathrm{CN}$ isomer, through intermediate states which are localized in the well at $\theta \approx \pi / 2 \mathrm{rad}$.

Let us conclude this section by pointing out that when the $\mathrm{CN}-\mathrm{Li}$ isomer is subjected to an electric field parallel to the $\mathrm{C} \leftarrow \mathrm{N}$ bond, as is the case here, the molecular dipole moment is mainly antiparallel to the electric field, and then the established torque will force the molecular rotation towards a more stable parallel configuration. If this happens, the assumed molecular orientation in the control Hamiltonian (2) changes, and then the rotationless vibrational Hamiltonian $H_{\mathrm{v}}$ in Eq. (1) is, in principle, no longer valid. However, we quantitatively show in Appendix A that this is not the case, since the controlled isomerization reaction is much faster than the molecular rotation.

\section{Quantum calculations}

To devise an effective strategy to control the $\mathrm{CN}-\mathrm{Li} / \mathrm{Li}-\mathrm{CN}$ isomerization reaction, we first need to know accurately the evolution of the LiCN vibrational energy spectrum as the electric field intensity increases. For this purpose, we use the discrete variable representationdistributed Gaussian basis method of Bačić and Light ${ }^{64}$ to compute the eigenenergies (and eigenstates) for the Hamiltonian operator corresponding to Eq. (2) for different values of the electric field intensity $\mathcal{E}$, since it is assumed that the electric field $\mathcal{E}$ is kept parallel to the $\mathrm{C} \leftarrow \mathrm{N}$ bond in the control process. In this way, the low 250 lying eigenfunctions $\langle R, \theta \mid n\rangle_{\mathcal{E}}, n=1, \ldots, 250$ were calculated for $\mathcal{E}=0,0.01,0.02, \ldots, 3\left(\times 10^{-3}\right.$ a.u. $)$, with their eigenenergies $E_{n}(\mathcal{E})$ converged to within $1 \mathrm{~cm}^{-1}$, being the 40 low lying eigenenergies (the most relevant in our study) converged to within $0.01 \mathrm{~cm}^{-1}$.

The second step, consisting the propagation in time of our initial state $|\psi\rangle_{t}$, as the electric field $\mathcal{E}(t)$ increases in time, is carried out by representing the quantum control Hamiltonian operator $\widehat{H}_{\mathrm{c}}(t)$ in Eq. (2) in the basis set of the 250 low lying eigenstates at zero field intensity $\left\{|n\rangle_{\mathcal{E}=0}\right\}_{n=1}^{250}$, that is, the eigenstates basis set of the vibrational Hamiltonian operator corresponding to Eq. (1), this leading to the following system of 250 ordinary differential equations

$$
i \hbar \dot{\mathrm{C}}=\mathrm{H}_{\mathrm{c}}(t) \cdot \mathrm{C},
$$

where $C$ is a column matrix containing the expansion coefficients $[\mathrm{C}]_{n}={ }_{0}\langle n \mid \psi\rangle_{t}$ of the evolving state $|\psi\rangle_{t}$, and $\mathrm{H}_{\mathrm{c}}(t)$ is the matrix representation of the control Hamiltonian operator

$$
\begin{aligned}
{\left[\mathrm{H}_{\mathrm{c}}(t)\right]_{m n} } & ={ }_{0}\left\langle m\left|\widehat{H}_{\mathrm{v}}-\widehat{\mathbf{p} \cdot \widehat{\mathcal{E}}(t)}\right| n\right\rangle_{0} \\
& =E_{n}(0) \delta_{m n}-{ }_{0}\left\langle m\left|\widehat{p}_{\|}\right| n\right\rangle_{0} \mathcal{E}(t),
\end{aligned}
$$

$E_{n}(0)$ being the eigenenergies at zero field, and $\widehat{p_{\|}}$the operator corresponding to the dipole moment component parallel to the $\mathrm{C} \leftarrow \mathrm{N}$ bond. The system of differential equations (4) was solved by using standard numerical methods, checking at each step the conservation of the wavefunction norm. We have also checked the suitability of the eigenstates basis set at zero field $\left\{|n\rangle_{0}\right\}_{n=1}^{250}$ in all the ranges of the calculations $\mathcal{E}=0-3 \cdot 10^{-3}$ a.u. by comparing the previously computed spectrum $E_{n}(\mathcal{E})$ and the diagonalization of the matrix representation $\mathrm{H}_{\mathrm{c}}$ at the same values of $\mathcal{E}$; this has always rendered a very good agreement for the relevant 40 low lying eigenstates.

\section{NAVIGATING THE SPECTRUM}

\section{A. Energy spectrum}

The correlation diagram of the vibrational energy levels $v s$. electric field intensity is depicted in Fig. 2. Obviously, since all states in this diagram belong to the same irreducible representation in the symmetry group of the effective potential energy function $V_{\text {eff }}(R, \theta)$, all crossings between states are in fact avoided (recall the Wigner-von Neumann non crossing rule ${ }^{65}$ ), although many of them are so narrow that appear as crossings to the naked eye.

As can be seen in the figure, all low lying eigenstates at $\mathcal{E}=0$ have a positive slope. This is due to the fact that they correspond to states in the $\mathrm{CN}-\mathrm{Li}$ isomer well, which interact positively with the field (given our assumed relative orientation of field and molecule). However, it is seen that these states present different values of the slope. In fact, those with smaller slopes, i.e., $n=1,2,3,4,6,7,9,11,12, \ldots$ correspond to a series with increasing excitation in $\theta$ and no excitation in $R$ coordinate. These states will be labeled $\left(n_{R}, n_{\theta}\right)_{\mathrm{CN}-\mathrm{Li}}=(0,0)$, $(0,1),(0,2),(0,3),(0,4),(0,5),(0,6),(0,7),(0,8), \ldots$ The series with steeper slopes, i.e., states $n=5,8,10,13, \ldots$ corresponds again to different excitations in the $\theta$ coordinate but with one excitation in the $R$ mode, that is, states $\left(n_{R}, n_{\theta}\right)_{\mathrm{Li}-\mathrm{CN}}=(1,0),(1,1),(1,2),(1,3), \ldots$. 


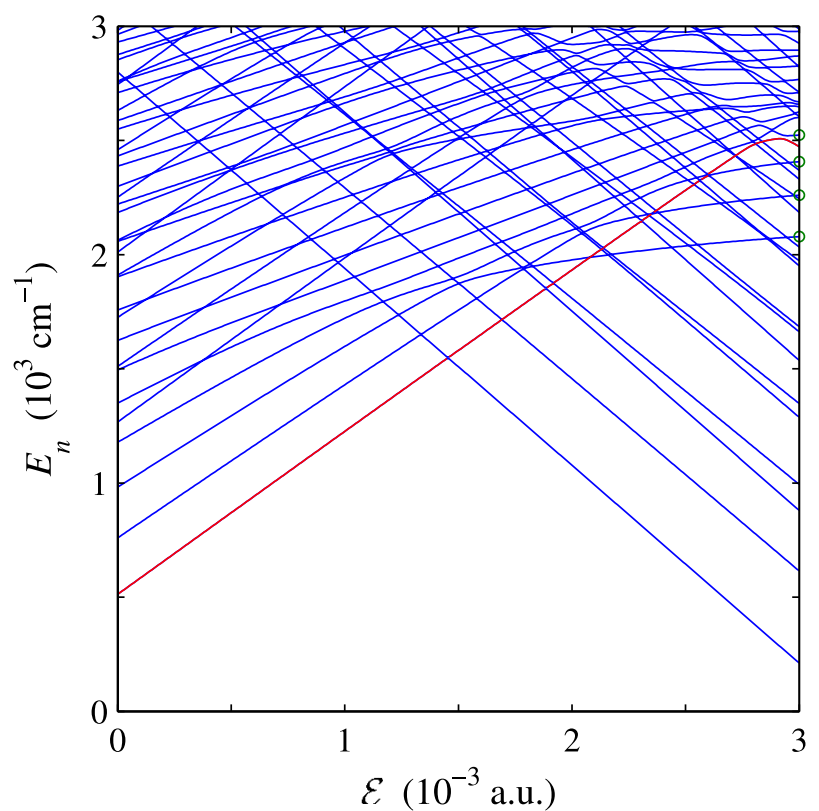

FIG. 2. Correlation diagram of eigenenergies (blue lines) vs. electric field intensity corresponding to the control Hamiltonian $\widehat{H}_{\mathrm{c}}$ of Eq. (2) with the electric field parallel to the $\mathrm{C} \leftarrow \mathrm{N}$ bond. The proposed navigation path from state $\left(n_{R}, n_{\theta}\right)_{\mathrm{CN}-\mathrm{Li}}=(0,0)$ in the $\mathrm{CN}-\mathrm{Li}$ isomer to state $(0,7)_{\mathrm{Li}-\mathrm{CN}}$ in $\mathrm{Li}-\mathrm{CN}$ is represented in red line. The four low lying states (represented in Fig. 3) localized in the well induced by the field at $\mathcal{E}=3 \cdot 10^{-3}$ a.u. [see Fig. 1(d)] are marked with green open circles.

On the other hand, at $\mathcal{E}=3 \cdot 10^{-3}$ a.u., all low lying eigenstates appear with a negative slope. They correspond to states in the $\mathrm{Li}-\mathrm{CN}$ well, which stabilizes (negative interaction) as the field increases. Similar to the previous case, states with smaller slopes (in absolute value), i.e., $n=1,2,4,6, \ldots$ have an increasing excitation in $\theta$ and no excitation in $R$ coordinate, that is, they correspond to states $\left(n_{R}, n_{\theta}\right)_{\mathrm{Li}-\mathrm{CN}}=(0,0),(0,1),(0,2),(0,3), \ldots$ And the series with steeper slopes (in absolute value) $n=3,5, \ldots$ corresponds to different excitations in the $\theta$ coordinate with only one excitation in $R$ coordinate, i.e., $\left(n_{R}, n_{\theta}\right)_{\mathrm{Li}-\mathrm{CN}}$ $=(1,0),(1,1), \ldots$.

Notice that the occurrence of broad avoided crossings (ACs) between states is very relevant at this point of the discussion. For example, states $n=8,9$ at $\mathcal{E}=3 \cdot 10^{-3}$ a.u. do so since they are involved in a 1:2 Fermi resonance, and then they are given by two orthogonal combinations of states $(0,4)_{\mathrm{Li}-\mathrm{CN}}$ and $(1,2)_{\mathrm{Li}-\mathrm{CN}}$, respectively. This topic has been discussed thoroughly in Ref. 66 and also in Ref. 51 in relation with the similar case of $\mathrm{KCN}$.

In addition to the above described states, there exists a third kind of eigenstates in the correlation diagram. They are those which appear localized in the well of $V_{\text {eff }}(R, \theta)$ at $\theta \approx \pi / 2 \mathrm{rad}$. They are induced by the electric field and correspond to a triangular configuration $\underset{\mathrm{CN}}{\mathrm{Li}}$. These states exhibit a small positive slope in the correlation diagram, as can be seen in Fig. 2, where the corresponding four low lying states of this kind at $\mathcal{E}=3 \cdot 10^{-3}$ a.u. (states $n=13$, $16,19,21)$ have been marked with green open circles. The associated probability densities $\langle R, \theta \mid n\rangle_{\mathcal{E}}$ are shown in Fig. 3, where it is seen that they correspond to increasing
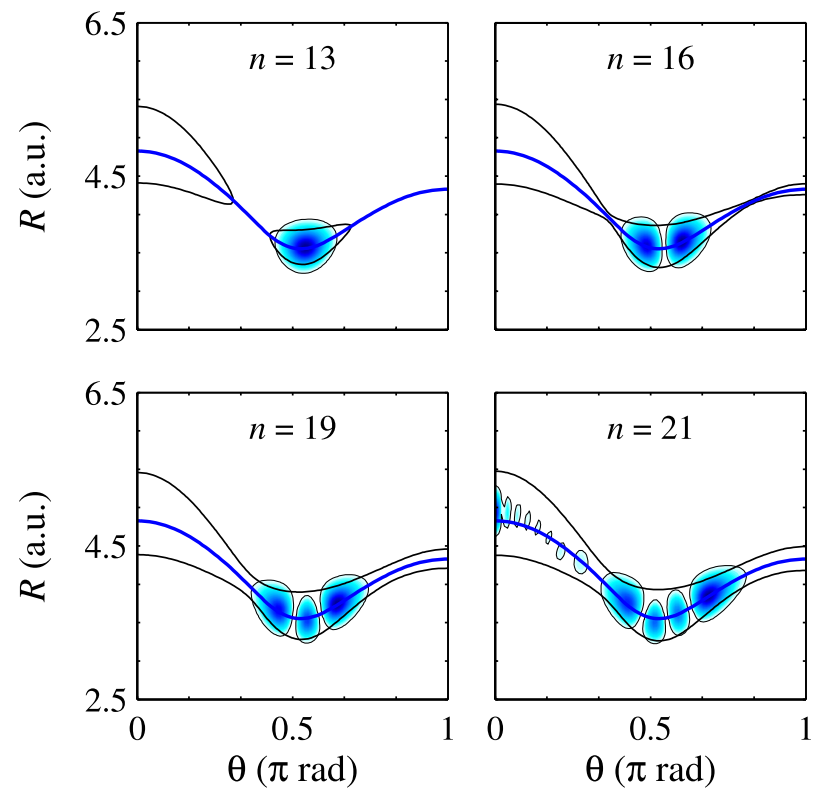

FIG. 3. Probability density for the four low lying eigenstates $\left(n_{R}, n_{\theta}\right)_{\Delta}$ $(0,0),(0,1),(0,2)$, and $(0,3)$ at $\mathcal{E}=3 \cdot 10^{-3}$ a.u., which are localized in the potential well induced by the field at $\theta \approx \pi / 2 \operatorname{rad}$ [see Fig. 1(d)]. Potential energy contours corresponding to each eigenenergy have been plotted in black lines. The minimum energy path is also included as a thick blue line.

excitations in the $\theta$ coordinate, so that they can be denoted as $\left(n_{R}, n_{\theta}\right)_{\Delta}=(0,0),(0,1),(0,2)$, and $(0,3)$, respectively. Notice here that, due to the proximity of the broad $\mathrm{AC}$ between states $(0,7)_{\mathrm{Li}-\mathrm{CN}}$ and $(0,3)_{\Delta}$, state $n=21$ is not a pure $(0,3)_{\triangle}$ state but it has a small contribution from state $(0,7)_{\mathrm{Li}-\mathrm{CN}}$, this being reflected in the existence of some density in the $\mathrm{Li}-\mathrm{CN}$ well with seven nodes.

\section{B. Navigation path}

To determine an adequate, or even optimal, navigation path in the energy spectrum, taking our system from a selected initial state to a desired final one, two important results have been taken into account as a guidance, namely, the adiabatic theorem ${ }^{67}$ and the Landau-Zener model, ${ }^{68,69}$ which establish the conditions for the navigation among the different curves of the correlation diagram, and quantify the transition probabilities at the different $\mathrm{ACs}$, depending on the rate $d \mathcal{E} / d t$ along the designed time profile $\mathcal{E}(t)$ (see Subsection III C).

In our case, we assume that the initial state for the controlled isomerization is the $\mathrm{CN}-\mathrm{Li}$ isomer ground state, i.e., $n=1$ at $\mathcal{E}=0$. Accordingly, it seems plausible in principle to increase the electric field intensity until reaching the neighborhood of the first $\mathrm{AC}$, and then, proceed with a slow enough rate $d \mathcal{E} / d t$ to adiabatically pass through it, thus getting to the ground state of the $\mathrm{Li}-\mathrm{CN}$ isomer. However, since the involved ACs are extremely narrow, the required rate for an adiabatic passage is excessively slow, and then inviable for all practical purposes. ${ }^{47}$

To realistically control isomerization, all excessively narrow ACs found in the path must be diabatically crossed, i.e., passed with a fast enough rate, while the broader ones can be overcome either adiabatically, i.e., traversed with a 
slow enough rate, or diabatically, i.e., with a fast rate. This should give the flexibility to navigate across the correlation diagram until reaching a state with $\mathrm{Li}-\mathrm{CN}$ characteristics. Accordingly, and using the above referenced rules, we obtained the navigation path indicated with a red line in Fig. 2 as one that can efficiently control the isomerization. In it, the target state corresponds to $(0,7)_{\mathrm{Li}-\mathrm{CN}}$ (state $n=20$ at $\mathcal{E}=3 \cdot 10^{-3}$ a.u.). Notice that in the region of broad ACs of the navigation path, i.e., $2.7 \cdot 10^{-3}<\mathcal{E}<3 \cdot 10^{-3}$ a.u., there also exist some narrow ACs. Then, this region must be passed with a rate which is slow enough for not crossing the broad ACs, but fast enough rate to cross these narrow ACs. Finally, also note that the state $(0,3) \triangle$ discussed above is involved in the navigation.

Notice that the target state is an exotic state which is not an eigenstate of free field Hamiltonian (1). Its actual shape will be discussed later in Sec. III D. Therefore, although this exotic state becomes unstable when the electric field is turned off, this may open up the possibility of performing new interesting chemistry, not possible otherwise.

\section{Electric field time profile}

After having fixed the navigation path in the energy spectrum, it is then necessary to establish an adequate electric field time profile $\mathcal{E}(t)$, i.e., one such that the probability $\left.\left.\right|_{3}\langle 20 \mid \psi\rangle_{t_{\mathrm{f}}}\right|^{2}$ of reaching the target (state $n=20$ at $\mathcal{E}=3 \cdot 10^{-3}$ a.u.) at the final time $t_{\mathrm{f}}$ is close to unity.

For this purpose, we follow the method previously described in Refs. 46 and 47, where a piecewise linear time profile $\mathcal{E}(t)$ is obtained in three steps: First, the relevant (broader) ACs in the chosen path are determined. Second, for each non-negligible AC that should be diabatically passed, we consider an instantaneous diabatic transition and find the locally optimal diabatic jump, i.e., we find the two field values, before and after the $\mathrm{AC}$, that maximize the overlap between the corresponding adiabatic states. And third, the time profile $\mathcal{E}(t)$ is linearly increased between the above mentioned diabatic jumps. Then, we start at $\mathcal{E}=0$ and proceed until the first non-negligible $\mathrm{AC}$ at $\mathcal{E}=2.035 \cdot 10^{-3}$ a.u. There a rate $d \mathcal{E} / d t$ is chosen in such a way that after this first piece of the profile, the overlap between the time evolved state and the adiabatic state is maximum. Once fixed the slope for the first non-diabatic step, the slope of the second slow piece (between the first two diabatic jumps) is optimized, again by maximizing the overlap with the final adiabatic state. Continuing this procedure, the remaining pieces of the time profile are successively determined in the same way.

By using this method, the time profile depicted in Fig. 4(a) was obtained. It consists of fifteen pieces and leads to a probability of reaching the final target state $\left.\left.\right|_{3}\langle 20 \mid \psi\rangle_{t_{\mathrm{f}}}\right|^{2}=0.974$ for $t_{\mathrm{f}}=14.27 \mathrm{ps}$. This straightforwardly calculated profile is nevertheless not exempt of problems, since as can be seen, it contains abrupt and vertical changes in the slope. Accordingly, it seems hardly, if at all, implementable in any actual experimental setup.

However, a time profile [blue line in Fig. 4(b)] more suitable for an experimental realization can be constructed by considering just two pieces in it. It consists of a first piece for

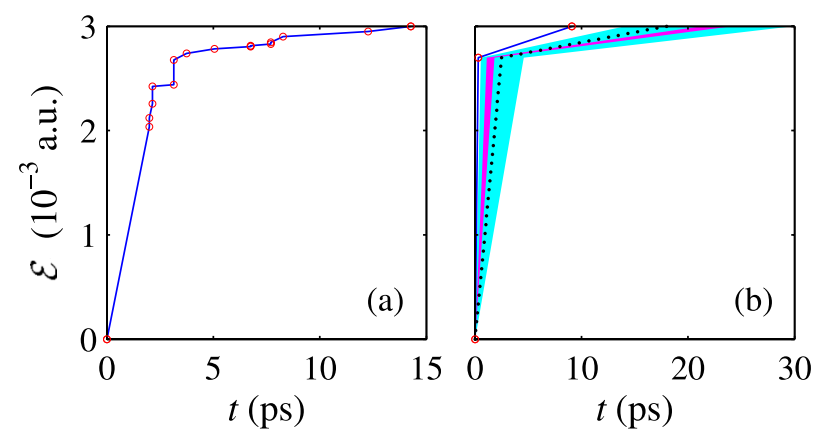

FIG. 4. (a) Linear piecewise electric field time profile for the control of the $\mathrm{CN}-\mathrm{Li} \leftrightarrows \mathrm{Li}-\mathrm{CN}$ isomerization reaction, consisting of fifteen different pieces obtained through local optimization of the transition probability at each avoided crossing in the navigation path. This leads to a probability of reaching the final target state $\left.\left.\right|_{3}\langle 20 \mid \psi\rangle_{t_{\mathrm{f}}}\right|^{2}=0.974$ for $t_{\mathrm{f}}=14.27 \mathrm{ps}$. (b) Same as (a) for a simpler electric field time profile (blue line) consisting of just two pieces, obtained by global optimization of the probability in the navigation path (see text for details). In this way, a final probability of 0.970 for $t_{\mathrm{f}}=9.1 \mathrm{ps}$ is obtained. Also shown in this panel are two cyan/magenta colored regions which indicate the ranges of still more simplified navigation profiles leading to a final probability always greater than $0.90 / 0.93$, respectively. Inside these ranges, the isomerization control can be considered as robust. An example of such robust navigation profiles, corresponding to a final probability of 0.940 for $t_{\mathrm{f}}=18 \mathrm{ps}$, is shown as a dotted line.

the range $0<\mathcal{E}<2.7 \cdot 10^{-3}$ a.u. with a large slope in order to diabatically pass all narrow ACs in the navigation path, and then a second one, for the range $2.7 \cdot 10^{-3}<\mathcal{E}<3 \cdot 10^{-3}$ a.u., which has a sufficiently small slope to adiabatically pass the broad ACs in the final part of the navigation. Besides, in the construction of this much simpler profile, a global (instead of local) optimization of the final probability was performed, by calculating the surface corresponding to the probability of reaching the final target state $\left.\left.\right|_{3}\langle 20 \mid \psi\rangle\right|^{2}$ as a function of the two involved piece time intervals $\Delta t_{1}$ and $\Delta t_{2}$. The results obtained in this way are presented in Fig. 5. They exhibit some features and peculiarities that are worth discussing.

In the first place, the whole surface in Fig. 5 exhibits a very wavelike shape that would be even more obvious in a three dimensional representation. However, we have chosen to use a contour plot since this is more useful for numerical purposes. This behavior can be understood in terms of the oscillatory behavior of the probability density as a function of time, as it is explained in Sec. III D. Second, the maximum value for the probability of reaching the final target state, i.e., the value corresponding to the optimum two-piece time profile, is surrounded by points with significantly lower values of that probability. This means that a small deviation from the optimum time profile in a hypothetical experimental realization will render a much poorer final result. Third, there exists a wide connected region in which the probability is greater than 0.90 , and also several smaller disconnected regions where the probability is even greater than 0.93 , as can be seen in the figure. The existence of these regions is very interesting from the experimental point of view, since it implies that any electric field time profile defined inside them render an approximately similarly good control of the isomerization process under consideration. In this sense, this control can be thought as being robust, as emphasized in the paper title. This means that sizable large deviations from the 


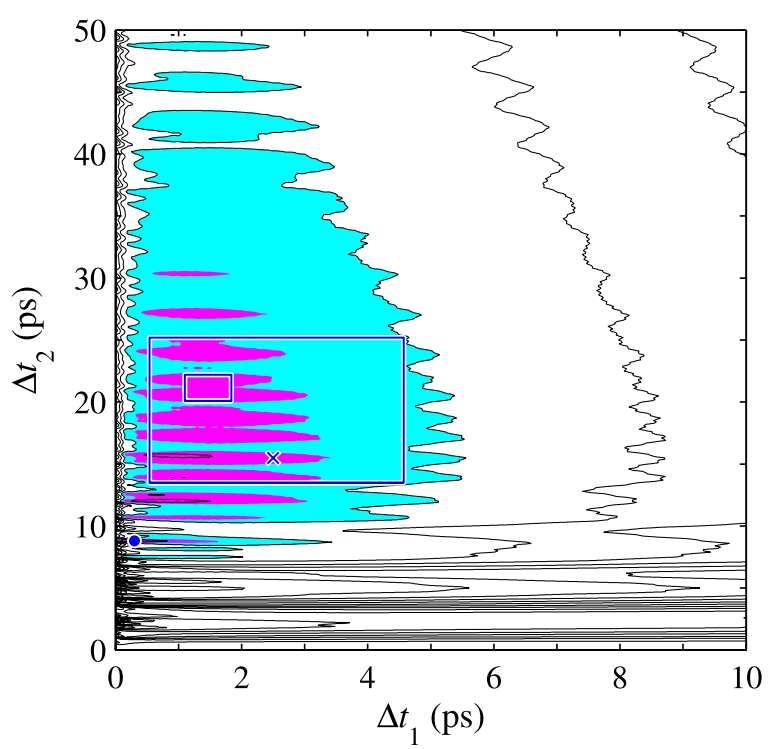

FIG. 5. Contour plot for the probability of reaching the final target state $\left.\left.\right|_{3}\langle 20 \mid \psi\rangle\right|^{2}$ in the $\mathrm{CN}-\mathrm{Li} \leftrightarrows \mathrm{Li}-\mathrm{CN}$ isomerization reaction controlled with the simple two pieces electric file profiles described in the text (see Sec. III C) as a function of the two time intervals $\Delta t_{1}$ and $\Delta t_{2}$ involved. Contours are spaced 0.05 . The cyan/magenta colored regions enclose the final probability ranges of $\left|{ }_{3}\langle 20 \mid \psi\rangle\right|^{2}=0.90-0.93 / 0.93-1$, and they correspond to the profiles marked with the same colors in Fig. 4(b). The position of the optimal result $\left.{ }_{3}\langle 20 \mid \psi\rangle\right|^{2}=0.974$ corresponding to the blue line profile in the same figure is marked with a blue filled circle. The regions of robust control corresponding to probabilities greater than 0.90 and 0.93 are indicated by the big and small rectangles, respectively. The robust electric field time profile giving a probability $\left.\left.\right|_{3}\langle 20 \mid \psi\rangle\right|^{2}=0.94$ [see dotted line in Fig. 4(b)] is indicated with a blue cross.

optimal time profile in an experimental realization will still lead to very good final results. Just for the sake of illustration, we have indicated two such (very simple) rectangular regions in Fig. 5. Any two-piece time profile defined inside the smaller one, which lies within the magenta colored area and also corresponds to the magenta colored region in Fig. 4(b), leads to a probability greater than 0.93 . Similarly, in the big rectangular region chosen within the cyan colored area (also corresponding to the cyan colored region in the time profile), any two-piece time profile would render a final probability greater than 0.90 . One example of such robust control behavior is that provided by the time profile represented with a dotted line in Fig. 4(b). Here, it is marked with a blue cross and leads to a probability of reaching the target state $\left.\left.\right|_{3}\langle 20 \mid \psi\rangle\right|^{2}=0.94$.

We close this part by stressing that although the slopes needed in the strong electric field profiles obtained with our controlling method are not small, they are within the reach of the available modern terahertz technology. ${ }^{43-45}$ Moreover, they can be however relaxed a bit and still get a good final probability of arriving at the desired target state, as demonstrated by the results of Fig. 5 .

\section{Probability density time evolution}

Of particular interest is to examine the time evolution of the probability density function $\left|\langle R, \theta \mid \psi\rangle_{t}\right|^{2}$ for the controlled isomerization process. For this purpose, we choose the robust two-piece time profile sample shown in dotted line in Fig. 4(b) (also the blue cross in Fig. 5). This is shown in Fig. 6, where the probability density is seen to change guided along the minimum energy path, from the $\mathrm{CN}-\mathrm{Li}$ isomer $(0,0)$ ground state at $t=0$ (also $\mathcal{E}=0$ ) to the mostly $\mathrm{Li}-\mathrm{CN}$ isomer $(0,7)$ state at $t=18$ ps (or $\mathcal{E}=3 \cdot 10^{-3}$ a.u.). In this process, the system passes through the ${ }_{\mathrm{CN}}^{\mathrm{Li}}$ intermediate $(0,3)_{\triangle}$ state at $t=13.2 \mathrm{ps}$ (or $\mathcal{E}=2.907 \cdot 10^{-3}$ a.u.) in the well at $\theta \approx \pi / 2 \mathrm{rad}$ induced by the field [see Sec. II $\mathrm{B}$ and Fig. 1(d)]. Moreover, it is worth noting that with the value chosen for the time step $(t=1.2 \mathrm{ps})$, the controlled isomerization process appears monotonic, with the probability density always flowing from the $\mathrm{CN}-\mathrm{Li}$ isomer to $\mathrm{Li}-\mathrm{CN}$, without never returning backwards. However, this is not the case and the flow really oscillates at a smaller scale in time, e.g., for a time step of $t=0.3 \mathrm{ps}^{70}$

Let us examine in more detail how the controlling mechanism is shown in the vibrational level spectrum. In the first part (piece) of the navigation path, i.e., from $\mathcal{E}=0$ to $\mathcal{E}=2.7 \cdot 10^{-3}$ a.u. (see red line in Fig. 2) using all the twopiece profiles of Fig. 4(b), there all (actually 15) narrow ACs are diabatically passed, and the isomerization process takes place monotonically. However, in the second part, i.e., from $\mathcal{E}=2.7 \cdot 10^{-3}$ a.u. to $\mathcal{E}=3 \cdot 10^{-3}$ a.u., two broad ACs are adiabatically passed and simultaneously four narrow ACs are diabatically crossed, and probability density indeed oscillates here in time back and forth between the two wells. This oscillating behavior can be understood in terms of the static
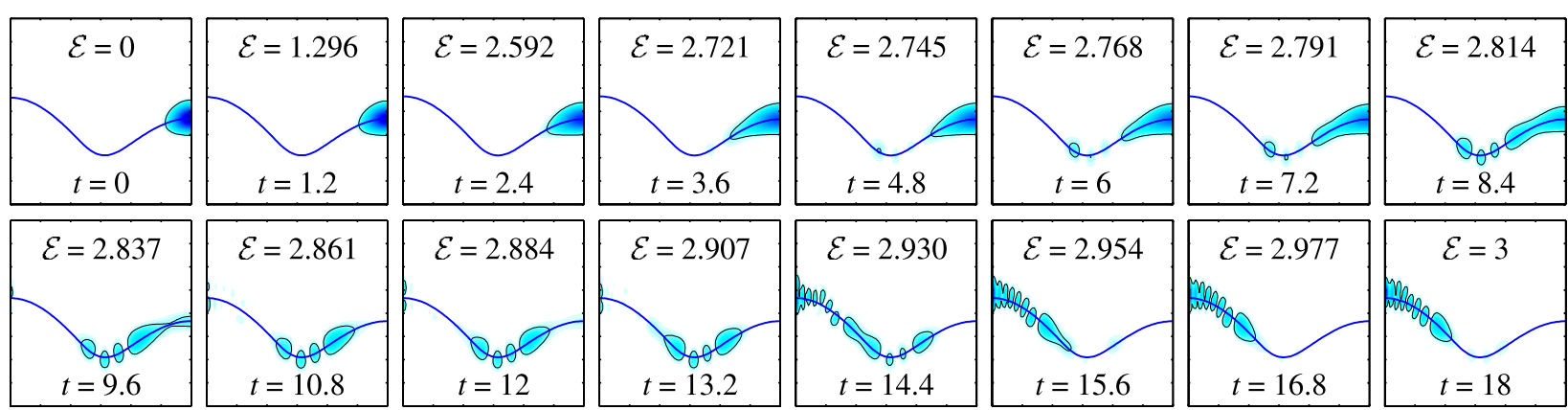

FIG. 6. Time evolution of the probability density function $\left|\langle R, \theta \mid \psi\rangle_{t}\right|^{2}$ corresponding to the control of the $\mathrm{CN}-\mathrm{Li} \leftrightarrows \mathrm{Li}-\mathrm{CN}$ isomerization reaction using the robust two-piece time profile sample in dotted line in Fig. 4(b). The time value (in ps) and the corresponding electric field amplitude $\mathcal{E}\left(\times 10^{-3}\right.$ a.u.) are indicated in each panel. The minimum energy path has also been plotted in blue thick line. The same nonlinear color scale has been used in all figures. Axes are the same as in Figs. 1 and 3. 
coupling, i.e., at fixed field, between the eigenstates involved in each two states AC. As is well known, the time evolution of the probability density $\left|\langle R, \theta \mid \phi\rangle_{t}\right|^{2}$ for a linear combination of two eigenstates $|\phi\rangle=c_{m}|m\rangle+c_{n}|n\rangle$ (with $\left|c_{m}\right|^{2}+\left|c_{n}\right|^{2}=1$ ) is given by

$$
\begin{aligned}
\left|\langle R, \theta \mid \phi\rangle_{t}\right|^{2}= & c_{m}^{2}|\langle R, \theta \mid m\rangle|^{2}+c_{n}^{2}|\langle R, \theta \mid n\rangle|^{2} \\
& +2 c_{m} c_{n}\langle R, \theta \mid m\rangle\langle n \mid R, \theta\rangle \cos \frac{\left(E_{m}-E_{n}\right) t}{\hbar},
\end{aligned}
$$

where $E_{m}$ and $E_{n}$ are the corresponding eigenenergies, and for the sake of simplicity, the eigenfunctions, $\langle R, \theta \mid m\rangle$ and $\langle R, \theta \mid m\rangle$, and their coefficients, $c_{m}$ and $c_{n}$, have been considered to be real-valued. Note that the probability density in Eq. (6) oscillates between the two extreme values: $\left|\langle R, \theta \mid \phi\rangle_{t}\right|^{2}=\left|c_{m}\langle R, \theta \mid m\rangle+c_{n}\langle R, \theta \mid n\rangle\right|^{2}$ for $t=\ell h /\left(E_{m}-E_{n}\right)$, with $\ell=0,1,2, \ldots$, and $\left|\langle R, \theta \mid \phi\rangle_{t}\right|^{2}$ $=\left|c_{m}\langle R, \theta \mid m\rangle-c_{n}\langle R, \theta \mid n\rangle\right|^{2}$ for $t=\left(\ell+\frac{1}{2}\right) h /\left(E_{m}-E_{n}\right)$. Let us recall again that in the second piece of the navigation path, the rate $d \mathcal{E} / d t$ of the field amplitude time profile must be fast enough to diabatically cross the narrow ACs. This implies that the broad ACs cannot be passed in a purely adiabatic manner. As a consequence, a little contribution from the upper eigenstate involved in each broad $\mathrm{AC}$ is picked up, this resulting in the oscillatory behavior predicted by the static approximation in Eq. (6). Furthermore, this oscillatory behavior is the reason for the wavelike shape of the surface represented in Fig. 5. Indeed, for the values of $\Delta t_{2}$ corresponding to the appropriate phases in the time evolution of the probability density, the probability of reaching the final target state results in local maxima, while for values of $\Delta t_{2}$ equal to the corresponding antiphases, the probabilities are locally minima, this giving rise to the wavelike shape of the surface.

\section{CONCLUDING REMARKS}

In this work, we report on the feasibility of an experimental realization for controlling isomerization using the technique of navigation in the spectrum introduced in Ref. 46. The application of this technique to the $\mathrm{CN}-\mathrm{Li} \leftrightarrows \mathrm{Li}-\mathrm{CN}$ (or similar) reaction was preliminary explored. ${ }^{47}$ Unfortunately, the approach used there required a rather complex electric field time profile, very demanding from the experimental point of view, and also left some open questions about the competition of the control with other simultaneous molecular processes.

We have shown here that our navigation control technique is robust, in the sense that there exist isomerizing electric field time profiles such that sizable large deviations from them still render very good final results. This is illustrated in the text by constructing a simplified version of the locally optimized ${ }^{46,47}$ fifteen pieces profile consisting of just two linear pieces, in which the optimization is performed globally. This simplified method exhibits a $97 \%$ of efficiency in bringing the system to the final desired target state when the two involved piece time intervals, $\Delta t_{1}$ and $\Delta t_{2}$, are optimized; but also we show wide convex regions of pairs $\left(\Delta t_{1}, \Delta t_{2}\right)$ where the efficiency is greater than, for example, 0.93 and 0.90 , respectively.
In our calculations, we have used a realistic model for LiCN, using analytical expressions fitted to ab initio quantum mechanical calculations for the potential energy surface ${ }^{49}$ and also for the dipole moment function. ${ }^{63}$ By using this realistic model, we have performed statistical classical simulations verifying that two main assumptions made in the work, namely, the rotationless motion assumed in the isomerizing system Hamiltonian and also the antiparallel orientation between electric field and molecular dipole moment, are completely reasonable.

Finally, it has been established that the oscillatory behavior existing in the time evolution of the probability density from the initial to the final state in the controlled isomerization process is due to the interaction between crossing states in the navigation path, thus leading to a wavelike structure in the probability surface for reaching the target state (see Fig. 5).

\section{ACKNOWLEDGMENTS}

This research was supported by the Ministry of Economy and Competitiveness-Spain under Contract Nos. MTM201239101 and ICMAT Severo Ochoa SEV-2001-0087, ANPCyTArgentina (PICT-1187), and CONICET-Argentina (PIP00273).

\section{APPENDIX A: MOLECULAR ROTATION DURING THE CONTROL PROCESS}

In order to check the validity of the two assumptions made in our proposed model to control the $\mathrm{CN}-\mathrm{Li} \leftrightarrows \mathrm{Li}-\mathrm{CN}$ isomerization reaction, i.e., rotationless approximation in Hamiltonian (1) and the antiparallel orientation between electric field and molecular dipole moment assumed in control Hamiltonian (2), made here and in Ref. 47, we have performed the classical calculations which are presented in this appendix.

The Hamiltonian function for our isomerizing system subjected to an external electric field $\mathcal{E}$ when including molecular rotation can be written explicitly as ${ }^{57}$

$$
\begin{aligned}
H= & \frac{P_{R}^{2}}{2 \mu_{1}}+\frac{P_{\theta_{R}}^{2}}{2 \mu_{1} R^{2}}+\frac{P_{\theta_{r}}^{2}}{2 \mu_{2} r_{\mathrm{eq}}^{2}}+V\left(R, \theta_{R}-\theta_{r}\right) \\
& -\left[p_{\|} \cos \left(\theta_{r}\right)-p_{\perp} \sin \left(\theta_{r}\right)\right] \mathcal{E}(t),
\end{aligned}
$$

where the new coordinates $\theta_{R}$ and $\theta_{r}$ are, respectively, the angles formed by the electric field $\mathcal{E}$ and the direction from the $\mathrm{C}-\mathrm{N}$ center of mass to the $\mathrm{Li}$ atom $\left(\begin{array}{l}\mathrm{C} \\ \mathrm{N}\end{array} \rightarrow \mathrm{Li}\right)$ and with the $\mathrm{C} \leftarrow \mathrm{N}$. Also, $p_{\|}(R, \theta)$ and $p_{\perp}(R, \theta)$ are the parallel and perpendicular components of the molecular dipole moment vector $\mathbf{p}(R, \theta)$ to the $\mathrm{C} \leftarrow \mathrm{N}$ bond, respectively, ${ }^{71}$ and $\mathcal{E}(t)$ is the time dependent electric field amplitude. Notice that the relationship existing between the angular coordinates in Eqs. (1) and (A1) is given by

$$
\theta=\theta_{R}-\theta_{r} .
$$

Using standard numerical methods, trajectories were propagated by integrating Hamilton's equations of motion corresponding to vibrorotational Hamiltonian (A1). In this calculation, both the fifteen-piece time electric field intensity 
profile and the simpler two-piece robust sample for the control of the above mentioned reaction, shown in Figs. 4(a) and 4(b), respectively (see Sec. III C), were used. A uniformly distributed ensemble of 10000 initial conditions $\left(P_{R}, P_{\theta_{R}}, P_{\theta_{r}}, R, \theta_{R}, \theta_{r}\right)_{0}$, with zero total angular momentum $P_{\theta_{R}}+P_{\theta_{r}}=0$, zero bond angle between $\mathcal{E}$ field and the $\mathrm{C} \leftarrow \mathrm{N} \theta_{r}=0$, and a total energy $H\left(P_{R}, P_{\theta_{R}}, P_{\theta_{r}}, R, \theta_{R}, \theta_{r}\right)$ $=512.44 \mathrm{~cm}^{-1}$, corresponding to the $\mathrm{CN}-\mathrm{Li}$ isomer ground state, were taken.

Results for the time evolution of these two ensembles, i.e., the two control field profiles, are shown in Figs. 7 and 8, respectively. In them we show, in the form of histograms, the distribution of the total angular momentum and alignment angle $\theta_{r}$ for different values of the evolution time. As can be seen, in both cases, the spreading of these two magnitudes is very small: $\Delta P= \pm 2.6 \cdot 10^{-3}$ a.u. for a final time $t=14.27 \mathrm{ps}$ and the fifteen pieces time profile in Fig. 4(a), and $\Delta P= \pm 3.4 \cdot 10^{-3}$ a.u. for a final time $t=18 \mathrm{ps}$ and the robust two-piece profile in Fig. 4(b). To get an idea of the magnitude of these numbers, we can consider, for comparison purposes, the maximum values of the angular momentum $P_{\theta}$ obtained in the dynamics of the rotationless Hamiltonian $H_{\mathrm{v}}$, which for the energy of the $\mathrm{CN}-\mathrm{Li}$ isomer ground state is equal to $P_{\theta}= \pm 14.2$ a.u. Moreover, the behavior of the alignment angle spreading is even better. For the fifteen pieces time profile, this spreading at final time $t=14.27 \mathrm{ps}$ amounts only to $\Delta \theta_{r}= \pm 4.7 \cdot 10^{-5} \pi \mathrm{rad}$, and for the robust two-piece time profile, the corresponding spreading at final time $t=18 \mathrm{ps}$ is $\Delta \theta_{r}= \pm 6.0 \cdot 10^{-5} \pi \mathrm{rad}$.

Summarizing, it can be stated that at the end of the controlled isomerization process, the rotationless approximation assumed in our model is good. Similarly, the orientation between field and $\mathrm{C}-\mathrm{N}$ bond direction remains fixed to a very good approximation throughout all the
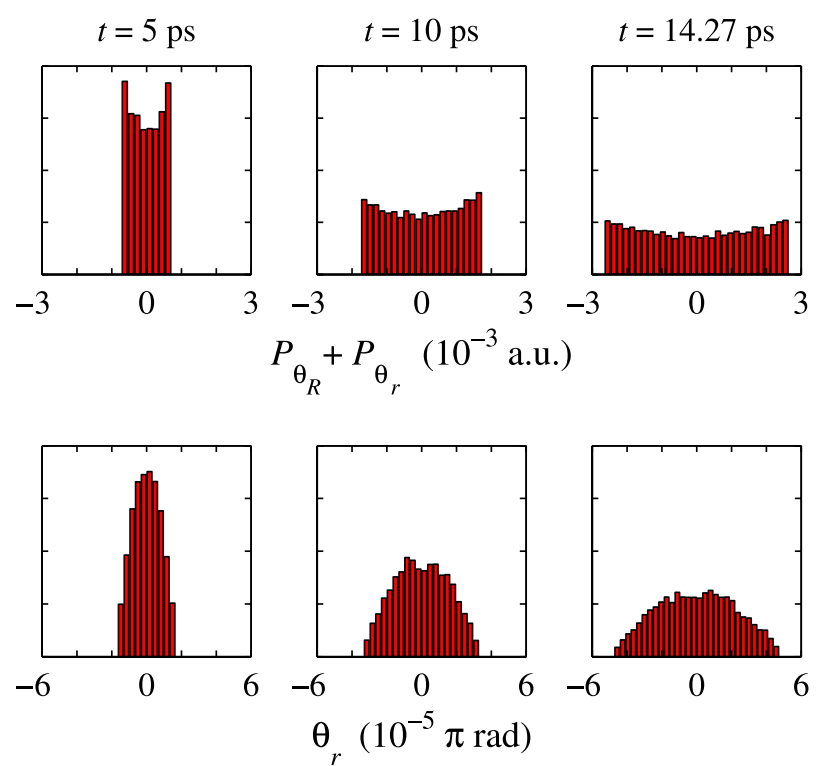

FIG. 7. Time evolution of the total angular momentum $P_{\theta_{R}}+P_{\theta_{r}}$ (top) and electric field $-\mathrm{C} \leftarrow \mathrm{N}$ bond alignment angle $\theta_{r}$ (bottom) for the control with the 15-piece electric field profile of Fig. 4(a). The distributions are initially located at $P_{\theta_{R}}+P_{\theta_{r}}=0$ and $\theta_{r}=0$, respectively, and the total energy corresponds to that of the $\mathrm{CN}-\mathrm{Li}$ isomer ground state.
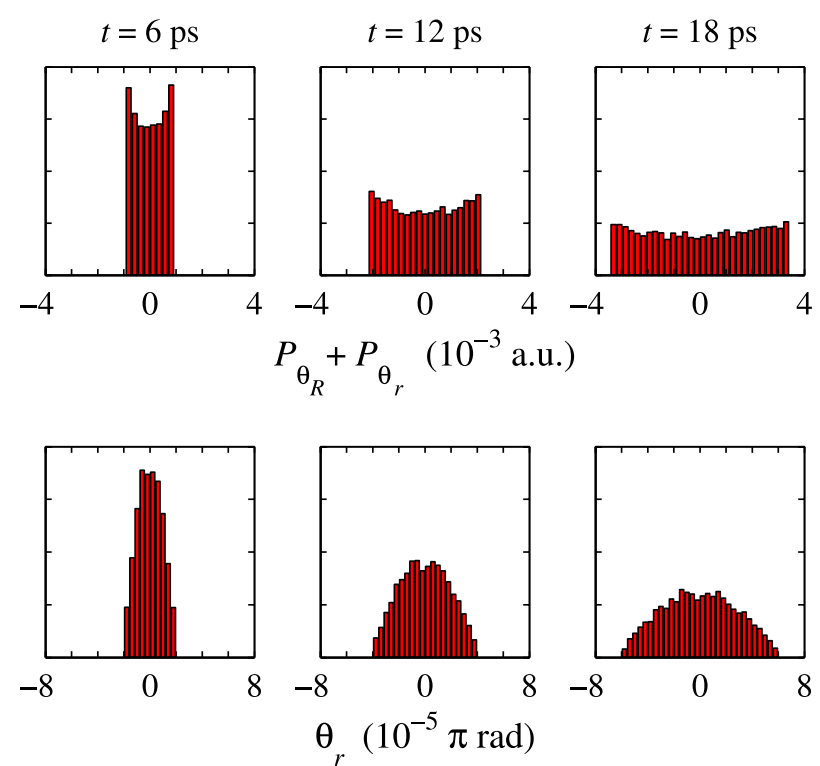

FIG. 8. Same as Fig. 7 for the control with the robust two-piece electric field profile of the dotted line in Fig. 4(b).

isomerization control process, as it is also assumed in our model.

\section{APPENDIX B: ELECTRIC FIELD—CN BOND MISALIGNMENT}

One of the simplifying assumptions made in our calculations is to consider that the controlling electric field and the $\mathrm{C} \leftarrow \mathrm{N}$ bond are perfectly aligned in a parallel fashion. Obviously, the experimental ability to achieve such perfect alignment is in fact limited. Actually, the typical accuracy obtained in representative experiments ${ }^{62}$ is estimated to be $\left\langle\cos ^{2} \theta_{r}\right\rangle=0.81$, corresponding to $\Delta \theta_{r}=25^{\circ}$. It is then pertinent to check here the robustness of our controlling technique against this inaccuracy; this being the aim of the present appendix.

Taking into account the results of Appendix A, the control Hamiltonian for the misaligned case can be written as

$$
\begin{aligned}
H_{\mathrm{c}}= & H_{\mathrm{v}}-\mathbf{p} \cdot \mathcal{E} \\
= & \frac{P_{R}^{2}}{2 \mu_{1}}+\left(\frac{1}{\mu_{1} R^{2}}+\frac{1}{\mu_{2} r_{\mathrm{eq}}^{2}}\right) \frac{P_{\theta}^{2}}{2}+V(R, \theta) \\
& -\left[p_{\|}(R, \theta) \cos \left(\theta_{r}\right)-p_{\perp}(R, \theta) \sin \left(\theta_{r}\right)\right] \mathcal{E}(t) .
\end{aligned}
$$

Accordingly, the matrix representation for this operator, required for the time evolution calculations in Eq. (4), is now given by

$$
\begin{aligned}
{\left[\mathrm{H}_{\mathrm{c}}(t)\right]_{m n}=} & { }_{0}\left\langle m\left|\left[\widehat{H}_{\mathrm{v}}-\widehat{\mathbf{p} \cdot \mathcal{E}(t)}\right]\right| n\right\rangle_{0} \\
= & E_{n}(0) \delta_{m n}-\left[{ }_{0}\left\langle m\left|\widehat{p}_{\|}\right| n\right\rangle_{0} \cos \left(\theta_{r}\right)\right. \\
& \left.-{ }_{0}\left\langle m\left|\widehat{p_{\perp}}\right| n\right\rangle_{0} \sin \left(\theta_{r}\right)\right] \mathcal{E}(t)
\end{aligned}
$$

instead of by Eq. (5). Let us remark here that the eigenstates $\left\{|n\rangle_{0}\right\}$ of the vibrational Hamiltonian constitute a complete basis set in the space with axial symmetry around the $\mathrm{C}-\mathrm{N}$ bond, corresponding to the aligned control Hamiltonian, but they are not a complete basis set in the space without that axial symmetry, this corresponding to the misaligned 

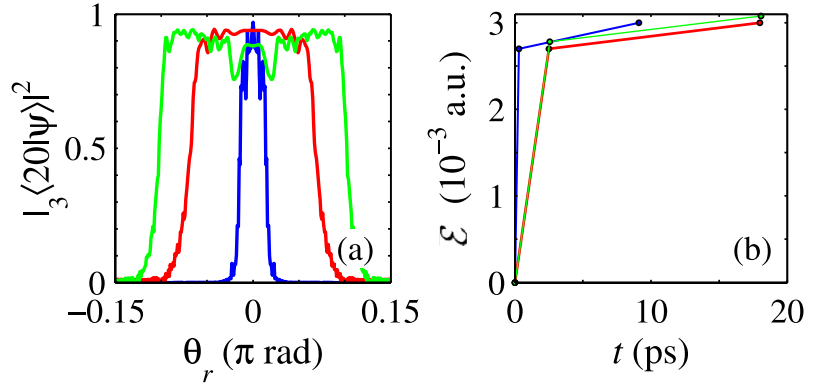

FIG. 9. (a) Probability of reaching the final target state $\left.\left.\right|_{3}\langle 20 \mid \psi\rangle\right|^{2}$ as a function of the alignment angle $\theta_{r}$ formed by the electric field $\mathcal{E}$ and the $\mathrm{C} \leftarrow \mathrm{N}$ direction. The blue line represents the values for the optimal profile (see the blue filled circle in Fig. 5), the red line is for the sample robust profile (see the blue cross in Fig. 5), and the green line indicates the results for a new controlling profile which guarantees high control efficiency in a range of misalignments closer to the typical uncertainties present in actual experiments ${ }^{62}$ (see the text for details). The corresponding time profiles for the controlling electric field are shown in panel (b).

control Hamiltonian. However, assuming small values for the misalignment, the calculations obtained by using the eigenstates $\left\{|n\rangle_{0}\right\}$ of the vibrational Hamiltonian can be considered as a good estimation of the true values.

Using Eq. (B2), we have calculated the probability of reaching the final target state $\left.\left.\right|_{3}\langle 20 \mid \psi\rangle\right|^{2}$ for different values of the angle $\theta_{r}$, considered as a parameter, and considering three different electric field time profiles: the first two correspond to cases discussed in Sec. III, i.e., the optimal profile corresponding to the blue filled circle in Fig. 5, and the sample robust profile corresponding to the blue cross in the same figure. The third one is a new time profile designed to guarantee control, i.e., high probability of reaching the desired target state in a range of misalignments close to the typical uncertainties present in actual experiments. ${ }^{62}$ This last profile is similar to the robust one, having the same two slopes, but the final electric field of each linear piece was slightly increased to compensate the contraction of the component in the $\mathrm{C} \leftarrow \mathrm{N}$ direction of the electric field due to misalignment. The results are shown in Fig. 9. As can be seen, when the robust time profile is used (red line), our method remains robust also against misalignment (e.g., it is fulfilled $\left.\left.\right|_{3}\langle 20 \mid \psi\rangle\right|^{2}>0.75$ in a range of $\left|\theta_{r}\right|<0.062 \pi \mathrm{rad}$, that is a misalignment of $\left.\pm 11.2^{\circ}\right)$. This range reduces however to $\left|\theta_{r}\right|<0.011 \pi \mathrm{rad}$ or $\pm 2^{\circ}$ when the optimal time profile (blue line) is used to control the reaction. Finally, when the new time profile in green is used, the range of robust control expands to $\left|\theta_{r}\right|<0.1 \pi \mathrm{rad}$ or $\pm 18^{\circ}$, which is fairly close to the experimental expected uncertainties.

${ }^{1}$ H. Rabitz, R. de Vivie-Riedle, M. Motzkus, and K. Kompa, Science 288, 824 (2000).

${ }^{2}$ R. S. Judson and H. Rabitz, Phys. Rev. Lett. 68, 1500 (1992).

${ }^{3}$ H. M. Wiseman and A. C. Doherty, Phys. Rev. Lett. 94, 070405 (2005).

${ }^{4}$ C. Ahn, A. C. Doherty, and A. J. Landahl, Phys. Rev. A 65, 042301 (2002).

${ }^{5}$ S. Mancini and H. M. Wiseman, Phys. Rev. A 75, 012330 (2007).

${ }^{6}$ I. Averbukh, J. Hepburn, V. Milner, and D. Tannor, eds., "Coherence and control in the quantum world," J. Phys. B: At. Mol. Opt. Phys. 48, 164000 (2015).

${ }^{7}$ A. M. Tyryshkin et al., Nat. Mater. 11, 143 (2011).

${ }^{8}$ M. Steger et al., Science 336, 1280 (2012).

${ }^{9}$ F. Shuang, A. Pechen, T. S. Ho, and H. Rabitz, J. Chem. Phys. 126, 134303 (2007).
${ }^{10}$ R. Romano and D. D'Alesandro, Phys. Rev. Lett. 97, 080402 (2006).

${ }^{11}$ F. Lucas and K. Hornberger, Phys. Rev. Lett. 113, 058301 (2014).

${ }^{12}$ G. Chen, D. A. Church, B. G. Englert, C. Henkel, B. Rohwedder, M. O. Scully, and M. S. Zubairy, Quantum Computing Devices: Principles, Designs, and Analysis (Chapman \& Hall/CRC, Boca Raton, 2007).

${ }^{13}$ J. Berezovsky, M. H. Mikkelsen, N. G. Stoltz, L. A. Coldren, and D. D. Awschalom, Science 320, 349 (2008).

${ }^{14}$ J. Hansom et al., Nat. Phys. 10, 725 (2014).

${ }^{15}$ D. Press, T. D. Ladd, B. Zhang, and Y. Yamamoto, Nature 456, 218 (2008).

${ }^{16} \mathrm{~S}$. Thiele, F. Balestro, R. Ballou, S. Klyatskaya, M. Ruben, and W. Wernsdorfer, Science 344, 1135 (2014).

${ }^{17}$ V. V. Dobrovitski, G. D. Fuchs, A. L. Falk, C. Santori, and D. D. Awschalom, Annu. Rev. Condens. Matter Phys. 4, 23 (2013).

${ }^{18}$ L. Childress et al., Science 314, 281 (2006).

${ }^{19}$ R. M. Stevenson, R. J. Young, P. Atkinson, K. Cooper, D. A. Ritchie, and A. J. Shields, Nature 439, 179 (2005).

${ }^{20}$ E. Peter, P. Senellart, D. Martrou, A. Lemaître, J. Hours, J. M. G'erard, and J. Bloch, Phys. Rev. Lett. 95, 067401 (2005).

${ }^{21}$ W. B. Gao, P. Fallahi, E. Togan, J. Miguel-Sanchez, and A. Imamoglu, Nature 491, 426 (2012)

${ }^{22}$ K. De Greve et al., Nature 491, 421 (2012).

${ }^{23}$ J. P. Reithmaier et al., Nature 432, 197 (2004).

${ }^{24} \mathrm{~K}$. Hennessy et al., Nature 445, 896 (2007).

${ }^{25}$ H. J. Krenner, M. Sabathil, E. C. Clark, A. Kress, D. Schuh, M. Bichler, G. Abstreiter, and J. J. Finley, Phys. Rev. Lett. 94, 057402 (2005).

${ }^{26}$ R. Hanson and D. D. Awschalom, Nature 453, 1043 (2008).

${ }^{27}$ D. Cao, H. O. Li, T. Tu, L. Wang, C. Zhou, M. Xiao, and G. P. Guo, Nat. Commun. 4, 1401 (2013)

${ }^{28} \mathrm{~N}$. Bloembergen and E. Yablonovitch, Phys. Today 31(5), 23 (1978).

${ }^{29}$ O. Skocek, C. Uiberacker, and W. Jakubetz, J. Phys. Chem. A 115, 7127 (2011).

${ }^{30}$ M. Gruebele, Theor. Chem. Acc. 109, 53 (2003).

${ }^{31}$ N. Elghobashi, P. Krause, J. Manz, and M. Oppel, Phys. Chem. Chem. Phys. 5, 4806 (2003)

${ }^{32}$ H. Lippert, J. Manz, M. Oppel, G. K. Paramonov, W. Radloff, H. H. Ritze, and V. Stert, Phys. Chem. Chem. Phys. 6, 4283 (2004).

${ }^{33}$ U. Gaubatz, P. Rudecki, S. Schiemann, and K. Bergmann, J. Chem. Phys. 92, 5363 (1990)

${ }^{34}$ Y. Kurosaki, T. S. Ho, and H. Rabitz, J. Chem. Phys. 140, 084305 (2014).

${ }^{35}$ S. A. Rice and M. Zhao, Optical Control of Molecular Dynamics (WileyInterscience, New York, 2000).

${ }^{36} \mathrm{M}$. Shapiro and P. Brumer, Principles of the Quantum Control of Molecular Processes (Wiley-Interscience, New York, 2003).

${ }^{37}$ C. Brif, R. Chakrabarti, and H. Rabitz, New J. Phys. 12, 075008 (2010).

${ }^{38}$ C. M. Tesch, K. L. Kompa, and R. de Vivie-Riedle, Chem. Phys. 267, 173 (2001).

${ }^{39}$ O. Kühn, J. Manz, and Y. Zhao, Phys. Chem. Chem. Phys. 1, 3103 (1999).

${ }^{40}$ S. Masuda and S. A. Rice, J. Phys. Chem. C 119, 14513 (2014).

${ }^{41}$ L. A. Pellouchoud and E. J. Reed, Phys. Rev. A 91, 052706 (2015).

${ }^{42}$ H. Petek, M. J. Weida, H. Nagano, and S. Ogawa, Science 288, 1402 (2000).

${ }^{43}$ H. Ogasawara, D. Nordlund, and A. Nilsson, FEL 2005, SLAC-PUB-11503 (2005).

${ }^{44}$ Z. Wu, A. S. Fisher, J. Goodfellow, M. Fuchs, D. Daranciang, M. Hogan, H. Loos, and A. Lindenberg, Rev. Sci. Instrum. 84, 022701 (2013).

${ }^{45}$ D. Daranciang, J. Goodfellow, M. Fuchs, H. Wen, S. Ghimire, D. A. Reis, H. Loos, A. S. Fisher, and A. M. Lindenberg, Appl. Phys. Lett. 99, 141117 (2011).

${ }^{46}$ G. E. Murgida, D. A. Wisniacki, and P. I. Tamborenea, Phys. Rev. Lett. 99, 036806 (2007)

${ }^{47}$ G. E. Murgida, D. A. Wisniacki, P. I. Tamborenea, and F. Borondo, Chem. Phys. Lett. 496, 356 (2010).

${ }^{48}$ D. Lee, I. S. Lim, Y. S. Lee, D. Hagebaum-Reignier, and G. Jeung, J. Chem. Phys. 126, 244313 (2007).

${ }^{49}$ R. Essers, J. Tennyson, and P. E. S. Wormer, Chem. Phys. Lett. 89, 223 (1982).

${ }^{50}$ J. N. Murrell, S. Carter, and L. O. Halonen, J. Mol. Spectrosc. 93, 307 (1982).

${ }^{51}$ H. Párraga, F. J. Arranz, R. M. Benito, and F. Borondo, J. Chem. Phys. 139, 194304 (2013).

${ }^{52}$ A. J. Marks, J. N. Murrell, and A. J. Stace, J. Chem. Phys. 94, 3908 (1991).

${ }^{53}$ H. Ishikawa, R. W. Field, S. C. Farantos, M. Joyeux, J. Koput, C. Beck, and R. Schinke, Annu. Rev. Phys. Chem. 50, 443 (1999).

${ }^{54}$ A. J. C. Varandas, J. Brandão, and L. A. M. Quintales, J. Phys. Chem. 92, 3732 (1988).

${ }^{55}$ A. Gamboa, H. Hernández, J. A. Ramilowski, J. C. Losada, R. M. Benito, F. Borondo, and D. Farrelly, Phys. Chem. Chem. Phys. 11, 8203 (2009). 
${ }^{56}$ P. L. Garcia-Muller, R. Hernandez, R. M. Benito, and F. Borondo, J. Chem. Phys. 141, 074312 (2014).

${ }^{57}$ R. Schinke, Photodissociation Dynamics (Cambridge University Press, Cambridge, 1993).

${ }^{58}$ P. L. Garcia-Muller, R. Hernandez, R. M. Benito, and F. Borondo, Phys. Rev. Lett. 101, 178302 (2008); J. Chem. Phys. 137, 204301 (2012).

${ }^{59}$ S. Fleischer, Y. Khodorkovsky, Y. Prior, and I. Sh. Averbukh, New J. Phys. 11, 105039 (2009).

${ }^{60}$ B. Friedrich and D. Herschbach, Phys. Rev. Lett. 74, 4623 (1995).

${ }^{61}$ M. Leibscher, I. Sh. Averbukh, and H. Rabitz, Phys. Rev. A 69, 013402 (2004).

${ }^{62}$ S. De, I. Znakovskaya, D. Ray, F. Anis, N. G. Johnson, I. A. Bocharova, M. Magrakvelidze, B. D. Esry, C. L. Cocke, I. V. Litvinyuk, and M. F. Kling, Phys. Rev. Lett. 103, 153002 (2009); 112, 159902 (2014).

${ }^{63}$ G. Brocks, J. Tennyson, and A. van der Avoird, J. Chem. Phys. 80, 3223 (1984). [Notice that there is a typo in this reference: The coefficients $a_{1,0}$ and $a_{3,0}$ for the dipole moment expansion of LiCN in Table II should be negative instead positive signed.]
${ }^{64}$ Z. Bačić and J. C. Light, J. Chem. Phys. 85, 4594 (1986).

${ }^{65} \mathrm{~J}$. von Neumann and E. P. Wigner, "Über das Verhalten von Eigenwerten bei adiabatischen Prozessen," in The Collected Works of Eugene Paul Wigner, edited by A. S. Wightman (1993), Vol. A, pp. 294-297.

${ }^{66}$ F. J. Arranz, F. Borondo, and R. M. Benito, J. Chem. Phys. 107, 2395 (1997).

${ }^{67}$ M. Born and V. Fock, Z. Phys. 51, 165 (1928).

${ }^{68}$ L. Landau, J. Phys. USSR 2, 46 (1932).

${ }^{69}$ C. Zener, Proc. R. Soc. A 137, 696 (1932).

${ }^{70}$ See supplementary material at http://dx.doi.org/10.1063/1.4936424 for an animation movie of the probability density function of the controlled isomerization process corresponding to the robust sample of twopiece time profile for equally spaced time intervals $t=0,0.3,0.6, \ldots$, 18 ps.

${ }^{71}$ Notice that in order to obtain these vector components, the orthogonal transformation $\left(\begin{array}{l}p_{\|} \\ p_{\perp}\end{array}\right)=\left(\begin{array}{c}\sin \theta \cos \theta \\ -\cos \theta \sin \theta\end{array}\right)\left(\begin{array}{l}\mu_{x} \\ \mu_{z}\end{array}\right)$ must be applied to the molecular dipole moment $\boldsymbol{\mu}=\left(\mu_{x}, \mu_{z}\right)$ defined in the Ref. 63 and used in the present work. 